\title{
REZENSIONEN
}

\section{Regierungssysteme der deutschen Bundesländer im Vergleich: Methodisch versiert, wenig problemorientiert}

Vatter, Adrian und Markus Freitag (Hrsg.): Die Demokratien der deutschen Bundesländer. Politische Institutionen im Vergleich. Mit einem Vorwort von Arend Lijphart, Opladen / Farmington Hills 2008, 355 Seiten, € 19,90.

Nachdem jahrzehntelang kaum jemand in der deutschen Politikwissenschaft sich mit den Regierungssystemen der Länder beschäftigen wollte, ist seit einigen Jahren ein regelrechter Boom in der Forschung zu verzeichnen. Der von Markus Freitag und Adrian Vatter herausgegebene Band unternimmt den ehrgeizigen Versuch, die wohl einflussreichste zeitgenössische Demokratiekonzeption - Arend Lijpharts Unterscheidung von Mehrheits- und Konsenssystemen - auf die gliedstaatliche Ebene der Bundesrepublik zu übertragen. Dass der „Meister“ selbst das Vorhaben durch ein Vorwort adelt, verwundert nicht, denn die Herausgeber und Autoren wissen sich nicht nur dessen typologischem Ansatz, sondern auch der methodischen Herangehensweise verpflichtet, die zur empirischen Überprüfung der Demokratieformen im wesentlichen auf sekundärstatistische Auswertungen zurückgreift. Das Lijphartsche Konzept wird allerdings nicht eins zu eins übernommen. Die umfangreiche und zugleich kritische Rezeption, die es in der Forschung erfahren hat, aufgreifend, nehmen die Autoren vielfältige Modifikationen vor, die einerseits dem besonderen Vergleichsgegenstand Rechnung tragen, andererseits der generellen Weiterentwicklung des Konzepts dienen. Insofern leisten sie einen substanziellen Beitrag zur typologischen Demokratieforschung.

Wichtige Änderungen ergeben sich bereits bei den für den Vergleich herangezogenen Kriterien. Da die deutschen Länder weder autonome Zentralbanken noch Zweite Kammern kennen, spielen diese Merkmale in der Adaption keine Rolle. Zur Überprüfung des bei Lijphart wesentlichen Föderalismus verwenden die Autoren stattdessen einen Dezentralisierungsindex, der die Ressourcenverteilung zwischen Ländern und Kommunen misst (S. 195 ff.). Unverzichtbar ist auch die Betrachtung der direktdemokratischen Institutionen, deren Nicht-Berücksichtigung beziehungsweise fehlerhafte Zuordnung eine der Hauptschwächen von Lijpharts Demokratiebuch darstellt.

Die Autoren weisen zu Recht darauf hin, dass die Regierungssysteme der Gliedstaaten geradezu optimale Voraussetzungen für den Systemvergleich mitbringen, da sie in ihren Hintergrundbedingungen in hohem Maße übereinstimmen (S. 17). Das Hauptaugenmerk der Untersuchung liegt entsprechend auf den institutionellen Unterschieden zwischen den 16 beziehungsweise - in der alten Bundesrepublik - elf Ländern, die auch in der zeitlichen Entwicklung detailliert herausgearbeitet werden. So viele interessante wie neuartige Befunde diese Herangehensweise zutage fördert, so sehr birgt sie allerdings auch die Gefahr, die stark ausgeprägte Homogenität der Regierungssysteme in den Ländern als Grundtatsache vergessen zu machen. Am Ende wird denn auch - eher beiläufig - eingeräumt, dass „es sich bei den Ländern um verschiedene Ausprägungsformen unterschiedlich stark dezen- 
tralisierter Demokratien (handelt), die auf der ersten Dimension der Lijphartschen Machtteilung zwischen schwach konsensualen und leicht majoritären Demokratien oszillieren “ (S. 322).

Auch an anderer Stelle verleitet das quantitativ-statistische Vorgehen dazu, dass man den sprichwörtlichen Wald vor lauter Bäumen nicht mehr sieht. So vermisst man im Kapitel über die Parteiensysteme zum Beispiel eine Erklärung für den doch erstaunlichen Befund, dass die Koalitionsbildung in den Ländern in den fünfziger Jahren - abweichend von derjenigen im Bund - mit zahlreichen Großen Koalitionen und Allparteienregierungen weitgehend dem konsensdemokratischen Muster folgte (S. 132 ff.). Im Kapitel über die Exekutiv-Legislativ-Beziehungen werden nicht nur die Änderungen im Zuge der jüngsten Föderalismusreform aus der Betrachtung ausgespart, sondern auch das Leerlaufen des parlamentarischen Regierungssystems auf der Beteiligungsebene des Bundesrates übersehen (S. 162 f.). Die Tatsache, dass es in den Ländern keine doppelte Exekutive gibt, wird hinsichtlich ihrer Konsequenzen für das parlamentarische System nicht näher erörtert, obwohl sie zum Beispiel das im nationalen Kontext ungewöhnliche Selbstauflösungsrecht sämtlicher Landesparlamente und das Nicht-Vorhandensein einer Vertrauensfrage in den meisten Ländern nach sich zieht (S. 164). Auch hätte man gerne gewusst, warum die Mehrzahl der Bundesländer inzwischen zu fünfährigen Wahlperioden übergegangen ist.

Unrichtig ist die Feststellung, dass den Landesverfassungen die klassische Montesquieusche Vorstellung der Gewaltenteilung zugrunde liege. Der Verfasserin des Kapitels (Martina Flick) ist offenbar nicht geläufig, dass eine Reihe von Ländern den so genannten neuen Dualismus durch die Einfügung von „Oppositionsklauseln“ in ihre Verfassungen inzwischen ausdrücklich normiert haben. Diese Klauseln waren gut gemeint, sind mit Blick auf das konsensdemokratische Format einer Minderheitsregierung aber problematisch, weil sie die verfassungsrechtliche Zuordnung einer Partei, die die Regierung toleriert, zur parlamentarischen Opposition in Frage stellt. Dass in einem Kapitel über die parlamentarische Regierungsform dieser mit Blick auf die Pluralisierung der Parteienlandschaft bedeutsamen Verfassungsreform keinerlei Beachtung geschenkt wird, ist merkwürdig. Bei aller verständlichen Fixierung auf Lijphart hätte den Autoren hier eine disziplinär etwas breiter angelegte Rezeption der Forschung gut getan.

Vor diesem Hintergrund klingt es ziemlich vermessen, wenn die Verfasser meinen, für ihren methodischen Ansatz einen höheren Anspruch reklamieren zu dürfen als die - so wörtlich - überwiegend „beschreibende(n) Darstellungen der parlamentarischen Systeme der deutschen Bundesländer und ihrer Institutionen“, die bisher vorlagen (S. 309). Bei Lichte betrachtet könnte es genau umgekehrt sein, mangelt es dem vorliegenden Buch doch an etwas, was gute Wissenschaft eigentlich auszeichnet: der Problemorientierung. Ist das Modell der Volksgesetzgebung im Rahmen eines parlamentarischen Regierungssystems praktikabel? Hat die parlamentarische Regierungsform selbst auf der Landesebene (noch) Zukunft, nachdem die Gliedstaaten im bundesdeutschen Föderalismus weniger mit Gesetzgebungs- als mit Verwaltungsaufgaben betraut sind? Wo könnten die Länder in institutioneller Hinsicht als Vorbild für den Bund dienen? Wer sich auf diese und andere Schlüsselfragen der „subnationalen Demokratien“ Antworten erhofft, wird das Buch nach der Lektüre von immerhin 327 Textseiten mit unzähligen Tabellen und Schaubildern enttäuscht oder verärgert zur Seite legen. In diesem Zusammenhang verwundert es dann nicht, dass Autoren wie Wilhelm Hennis oder Theodor Eschenburg, die in den fünfziger und sechziger Jahren wegweisende politologische Analysen zu den Regierungssystemen der Länder 
vorgelegt haben, im Literaturverzeichnis gar nicht mehr auftauchen - genauso wenig wie der streitbare und gerade deshalb einschlägige Hans Herbert von Arnim. Freitags und Vatters Sammelwerk stellt gewiss eine beeindruckende Forschungsleistung dar, deren komparativstatistischer Zugriff unsere Kenntnisse der deutschen Länderregierungssysteme nennenswert bereichert. Wer das ganze Bild will, wird aber weiterhin (auch) zu anderen Quellen greifen müssen.

Frank Decker

\section{Ex oriente lux? Der Beitrag der fünf neuen Länder zur direkten Demokratie auf dem Prüfstand}

Neumann, Peter: Sachunmittelbare Demokratie im Bundes- und Landesverfassungsrecht unter besonderer Berücksichtigung der neuen Länder, Nomos Verlagsgesellschaft, Baden-Baden 2009, 999 Seiten, €149,-.

Nach einer Erläuterung des Forschungsstandes gibt Neumann einen Überblick, wie sich die Debatte um die plebiszitären Elemente in der Bundesrepublik Deutschland von den Anfängen bis heute entwickelt hat, wozu später ein Kapitel über unmittelbare Demokratie im Verfassungsrecht der DDR gehört. Es folgen eine Analyse der Instrumente unmittelbarer Demokratie auf Verfassungsebene in der Bundesrepublik Deutschland und eine eingehende Auseinandersetzung mit den grundgesetzlichen Grenzen der Verfassungsgebung in den Ländern. Diese wird insbesondere für die ostdeutschen Länder in eigenen Kapiteln untersucht, wobei speziell auf die sachunmittelbare Demokratie in den Landesverfassungen und auch auf die praktischen Erfahrungen, die man in Brandenburg, Mecklenburg-Vorpommern, Sachsen, Sachsen-Anhalt und Thüringen damit gemacht hat, eingegangen wird. Am Ende gibt der Autor Empfehlungen für die Reform des Landesverfassungsrechts (nicht nur) der neuen Länder de constitutione ferenda, ausgearbeitet in einem konkreten Entwurf, und schließt mit nützlichen Übersichten und Tabellen sowie Stichwort- und Personenverzeichnissen.

Qualitativ kann man bei dem voluminösen Werk zwei Schichten unterscheiden. Die Untersuchung zum Beispiel des Rechtscharakters der 1989 in Schleswig-Holstein entwickelten und in den Verfassungen von elf Ländern, darunter alle neuen, rezipierten „Volksinitiative“, der je nach dem systematischen Zusammenhang variiert (S. 189 - 205), zeugt von dogmatischem Scharfsinn. Auch die Analysen, ob Quoren beziehungsweise Finanzvorbehalte wirklich notwendige Bedingungen der Volksgesetzgebung sind (S. $344-439$ ), erfolgen glänzend; Neumanns negative Antwort überzeugt. Quoren etwa sind nicht einmal für verfassungsändernde Volksentscheide rechtsstaatlich geboten, denn die Volksgesetzgebung ist per se ein erschwertes Gesetzgebungsverfahren (S. 366 - 370). Wie Neumann hier mit Staatsrechtslehrern von Josef Isensee bis Hans-Detlef Horn die Klinge kreuzt, ist ein Genuss zu lesen. Die 216 Seiten über die sachunmittelbare Demokratie in den Verfassungen der neuen Länder hingegen, in denen er etwa alle 37 Entwürfe, die vor oder während der Verfassungsgebung vorgelegt wurden, und ihre parlamentarische Behandlung darstellt, gefolgt von weiteren 158 Seiten über die Staatspraxis, bei der der formale Verfahrensablauf von 80 Initiativen, 16 Volksbegehren und zwei Volksentscheiden beschrieben wird, 